\title{
A little incoherence to improve the clarity
}

\author{
Murray Hamilton \\ Department of Physics and Mathematical Physics, The University of Adelaide, Adelaide SA \\ 5005, Australia
}

\begin{abstract}
Interference resulting from the measurement of four-time fourth-order correlation functions of a wave field is discussed. The fringes that are observed are peculiar because they have a statistical origin, and show the greatest contrast when the coherence time of the field is finite. This is demonstrated with a simple acoustic experiment. Random telegraph phase noise is used in this experiment to vary the field coherence in order to highlight the problem of interpreting this interference; for this noise the Gaussian moment theorem may not be invoked to reduce the description of the interference to one in terms of first order interference. A second example using the pseudorandom phase fluctuations that are encoded on GPS satellite transmissions is also presented.
\end{abstract}

Keywords: coherence, interference, second order interference

\section{INTRODUCTION}

It is well established that the familiar interference that is observed in Michelson interferometers is described by the autocorrelation function of the wave field, $G^{(2)}(t ; 0)=\left\langle E^{*}(t) E(0)\right\rangle$. However, just as this is the first in a hierarchy of correlation orders, so also is the interference just the first in a hierarchy of orders. Second order interference results when the signal depends on a fourth order correlation function. The standard example is the intensity autocorrelation function $G^{(4)}(0, t ; t, 0)=\left\langle E^{*}(0) E^{*}(t) E(t) E(0)\right\rangle=\langle I(t) I(0)\rangle$, which is a two-time, fourth-order correlation of the field. It was measured in the classic optical experiment by Hanbury-Brown and Twiss, ${ }^{1}$ where a beam was split into two parts and the rate of photon coincidences detected between the two beams was measured. We are concerned here with a more general fourth order correlation of the field, the four time correlation function $G^{(4)}(t, t+\tau ; \tau, 0)=\left\langle E^{*}(t) E^{*}(t+\tau) E(\tau) E(0)\right\rangle$. One of the time arguments is taken to be zero because the stochastic processes involved are assumed to be stationary and ergodicity is assumed. The times $t$ and $\tau$ are mathematically equivalent, but physically one is a variable parameter of the experiment (a delay time) whereas the other is the usual time variable. Nonlinear optical experiments can be set up that measure the Fourier transform of such functions. For example, in a two photon absorption experiment that incorporates a retroreflecting mirror, ${ }^{2}$ so that the laser beam is passed twice through the absorber, the two-photon absorption rate is proportional to the Fourier transform of $G^{(4)}(t, t+\tau ; \tau, 0) .^{3}$ Also, some four-wave mixing and coherent Raman spectroscopy experiments ${ }^{4-6}$ measure the Fourier transform of four-time sixth-order field correlation functions. Alternatively, for low frequency fields such as acoustic or radio waves, direct detection of the fields, followed by computation of the correlation, is possible. Although I describe acoustic and radio experiments in this paper, the inspiration for this work actually comes from nonlinear optics and the theoretical ideas expressed in this paper apply to any scalar wave field.

A very interesting feature of the measurements of these higher order correlation functions is the appearance of second order interference fringes (although in the case of two photon absorption this remains a prediction). As I shall explain below, these fringes have a statistical origin which is quite unlike the ordinary first order interference phenomena seen in a Michelson interferometer, for example. The fringes are particularly interesting because they show greatest contrast when the fields are partially first order coherent, rather than fully coherent or incoherent. This work was first published in a previous paper ${ }^{7}$; here I expand on that paper and present a second example.

In measurements of the two time correlation function $G^{(4)}(0, t ; t, 0)$, interference fringes have been observed, but this requires uniquely quantum mechanical light fields with pairs of correlated and indistinguishable photons, ${ }^{8}$

Further author information: E-mail: murray.hamilton@adelaide.edu.au 


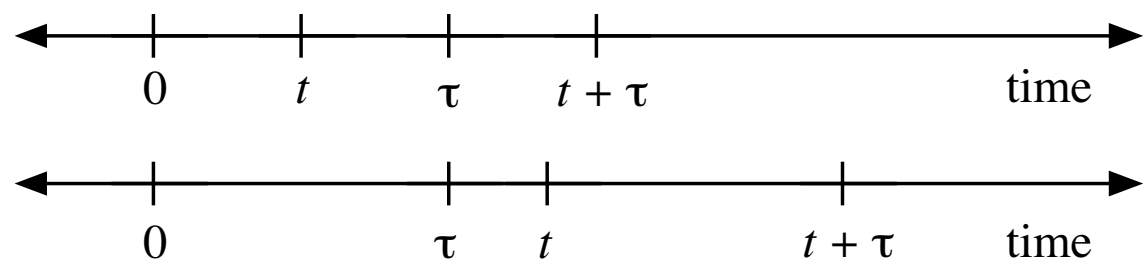

Figure 1. Two different time regimes must be considered for the evaluation of $G^{(4)}(t, t+\tau ; \tau, 0)$, leading to a sharp corner in this function. In (a), where $t<\tau$ there are three time intervals in which the fluctuations in the field can be considered statistically independent $[0, t),[t, \tau),[\tau, t+\tau]$. However if $t>\tau$ the set of intervals shown in (a) ceases to be statistically independent and we must consider the intervals shown in (b), i.e., $[0, \tau),[\tau, t),[t, t+\tau]$. The correlation must be calculated separately for the cases (a) and (b).

or pairs of coherently excited atoms. ${ }^{9}$ In these cases it can be arranged that no first order interference is observed, even though second order fringes are manifest. Nevertheless, in these experiments the second order fringes can be described in terms of first order interference of quantum probability amplitudes. For the moment though, we consider only fields that can be represented classically and have some associated stochastic process which makes them partially coherent. It is also important to note also that we are ignoring spatial correlations in what follows. If the intensity spatial autocorrelation function is considered, second order interference fringes may be observed if the field has underlying first order interference fringes ${ }^{10,11}$ With strictly classical fields, one normally would not expect to see interference fringes in the temporal correlation $G^{(4)}(0, t ; t, 0)$ because each of the two physical fields is measured by an intensity detector; the direct separate measurement of intensity destroys information about the phase difference between the fields.

With four time correlation functions however, one can see interference fringes with purely classical fields. The idea behind this is illustrated in Fig. 1. The four time nature of the correlation functions leads to a sharp corner in the correlation function, i.e. the derivative is discontinuous at some time $t$, as long as there is a stochastic process to introduce some incoherence. Then the Fourier transform of the correlation function will have oscillations that we interpret as second order interference fringes. The existence of these fringes depends on the stochastic process; if it were absent, the correlation function would be a product of sinusoids, whose Fourier transform is just a sum of delta functions.

In what follows we consider manifestations of second order fringes in spectroscopy in more detail (section 2), in order to introduce a possible interpretation of higher order interference in terms of first order interference. We shall show that this approach lacks generality however. An important conclusion of this work is that the fringes that arise from four time correlations cannot, in general, be explained in terms of a description in terms of first order interference. Then in section 3 we consider an actual experiment in which this lack of generality is highlighted by a suitable choice of stochastic process to make the field partially coherent. A second example (a simulation) using GPS transmissions is described in the following section, which points at possible applications of this interference in ranging. Finally we consider some issues concerning spatial coherence and quantum mechanical fields which lie somewhat to the side of the main direction of this paper but are still part of the overall context.

\section{SECOND ORDER FRINGES IN SPECTROSCOPY}

To give these ideas more concreteness, consider a spectroscopic two-photon absorption experiment, with an atomic vapour and incorporating a retroreflecting mirror, ${ }^{2}$ so that the laser beam is passed twice through the absorber. This is done to make the measurement Doppler-free; if a photon is aborbed from each of the counterpropagating beams by a single atom, the Doppler shifts due to movement of the atom along the beam axis cancel. In the limit where the linewidth of the two-photon transition is very small, the absorption rate is 
proportional to the Fourier transform of $G^{(4)}(t, t+\tau ; \tau, 0),^{3}$

$$
W(\omega)=\int_{-\infty}^{\infty} \exp (-i \omega t) G^{(4)}(t, t+\tau ; \tau, 0) d t
$$

Here $\tau$ is the time delay that the light suffers in going from the absorber to the retroreflecting mirror and back again. Now there is only one detector, the two-photon absorber, so the relative phase information of the fields is preserved. If one were to monitor the value of $W(\omega)$ at a given frequency $\omega$, one would see this value oscillate as the delay time is varied. An operational definition of what constitutes interference fringes would be such oscillations of a signal; that is, as one field is delayed with respect to the other. This is similar to a Michelson interferometer, though the phase of the field plays a different role here. Alternatively, it can be more practical to simply observe the oscillations in the spectrum $W(\omega)$ itself, for constant $\tau$.

One way of interpreting such oscillations presents itself if the stochastic process that underlies any lack of coherence in the wave field has a Gaussian density function. This is to appeal to the Gaussian moment theorem ${ }^{12}$ whereby the higher order correlation function is reduced to the sum of products of second order correlations. For the case of the four-time correlation that we are concerned with, this reads

$$
\left\langle E^{*}(t) E^{*}(t+\tau) E(\tau) E(0)\right\rangle=\left\langle E^{*}(t) E(\tau)\right\rangle\left\langle E^{*}(t+\tau) E(0)\right\rangle+\left\langle E^{*}(t) E(0)\right\rangle\left\langle E^{*}(t+\tau) E(\tau)\right\rangle .
$$

For this class of stochastic process, it is possible to explain the second order fringes in terms of first order interferences, rather than resorting to an explanation in the style of Fig. 1.

As noted above, higher order fringes have been observed in four-wave mixing and coherent Raman spectroscopy experiments ${ }^{4-6}$ where the signal was proportional to the Fourier transform of a four-time sixth-order correlation function. In the signal, third order interference fringes were seen and were termed radiation difference oscillations. These were interpreted by appealing to the Gaussian moment theorem, which was possible since in that work pulsed dye lasers were used. It was reasonable to assume that the underlying stochastic process had a Gaussian density function, because the independent fluctuations in the many laser modes that are present give rise to Gaussian statistics according to the central limit theorem. Thus the sixth order correlations were reduced to products of second order correlation functions; that is, of first order interferences.

However such an interpretation is not possible if the stochastic process is non-Gaussian, which is why we have chosen a random telegraph process ${ }^{13}$ with a two-peaked density function for the acoustic experiment described below. In a random telegraph process the noisy variable switches randomly between two well defined values and the number of phase transitions in a given time is a Poisson random variable. The average time that the variable spends at either of the values is the dwell time $T$. If the noise is applied to the phase of the field and the size of the phase jumps is $\pi, T / 2$ can be identified with the coherence time. Further, previous experiments have not demonstrated that the fringes that arise from four time correlations have maximum contrast if the field is partially coherent; that is, their existence requires the existence of the stochastic process, as suggested by Fig. 1.

\section{ACOUSTIC EXPERIMENT}

As noted above, relatively low frequency waves such as acoustic or radio waves, allow one to measure directly each of the fields simultaneously and then compute $W(\omega)$ which is defined by equation 1 . If there is only one source of the field, one could of course measure time series data for the field at just one place and still compute $W(\omega)$. However we choose to measure the field from a single source at two places, which is equivalent to two times. This allows the second order fringes to be used to measure the difference in the distances between two points and the source, provided the wave speed is known. Thus the the fact that the periodicity of the fringes is determined by the delay $\tau$ suggests that measuring the Fourier transform of $G^{(4)}(t, t+\tau ; \tau, 0)$ might have some utility as a ranging technique.

To test the prediction that the second order fringes have maximum contrast when the field coherence time is finite, a speaker and two microphones were set up in an anechoic chamber such that the distance from the speaker to each of the two microphones differed by $50 \mathrm{~cm}$. The speaker was a moving-coil dome "tweeter" 


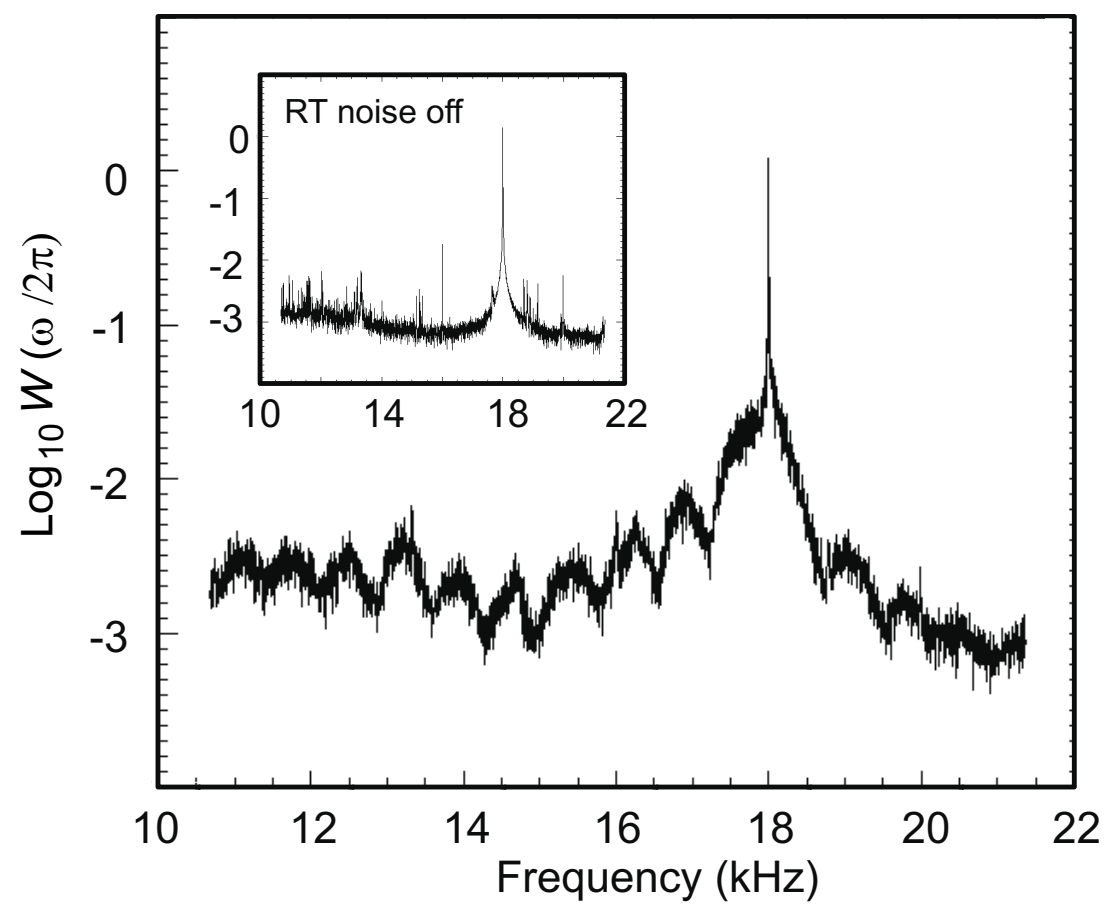

Figure 2. The Fourier transform of $G^{(4)}(t, t+\tau ; \tau, 0), W(\omega)$, for $\tau=1.34 \mathrm{~ms}, T=34 \mathrm{~ms}$ and $\phi=\pi$. The transform shown is the average of four transforms, each using $32 \mathrm{k}$ samples. The peak at $18 \mathrm{kHz}$ is due to the $9 \mathrm{kHz}$ frequency of the sound. The second order interference fringes are the oscillations on the wings of this peak. The inset shows $W(\omega)$ when the random telegraph phase modulation is switched off.

(Digitor model M98623) with a frequency range from $1.5 \mathrm{kHz}$ to $20 \mathrm{kHz}$ and RMS input power rating of $5 \mathrm{~W}$. The microphones were both simple electret microphones. The sinusoidal signal from a function generator (Hewlett Packard HP3325) was applied to the speaker with a frequency of $9 \mathrm{kHz}$ and an amplitude of $40 \mathrm{mV}$ at the speaker (nominal impedance $8 \Omega$ ). Applied to the signal, via the phase modulation input of the HP3325, was a random telegraph signal of variable dwell time $T$. The size $\phi$ of the phase jump was also variable. This modulation signal was produced by a voltage noise generator that has been previously described in the literature. ${ }^{14}$ The two microphone signals were amplified (Tektronix 7A22 amplifiers) and recorded simultaneously at $50 \mathrm{kS} / \mathrm{s}$ and 12-bit resolution. From the recorded microphone signals $W(\omega)$ was calculated. The delay time $\tau$ is now the time that the sound takes to travel the $50 \mathrm{~cm}$ spacing between the microphones. This calculation is speeded up by noting that we in fact calculate the power spectrum of the product of fields $H(t, \tau)=E(t) E(t+\tau)$; this is just the modulus squared of the transform of $H(t, \tau)$ which is rapidly computed with a standard fast Fourier transform algorithm. Fig. 2 shows a part of one of these spectra. The oscillations seen in the wings of the spectrum are the second-order fringes that we seek.

The spacing of the fringes in Fig. 2 is $720 \pm 20 \mathrm{~Hz}$ which is in reasonable agreement with the reciprocal of the delay time $\tau=1.43 \mathrm{~ms}$, as expected from the theory. ${ }^{15}$ It can be seen that the peak at $18 \mathrm{kHz}$ is somewhat asymmetric; the fringes are much less visible on the high frequency side. For $\pi$ radian phase jumps the spectrum has the form of a Lorentzian with modulated wings ${ }^{15}$;

$$
W(\omega)=-\frac{\exp (-4 \tau / T)}{\Delta} \sin (\Delta \tau)+\frac{4 / T}{(4 / T)^{2}-\Delta^{2}}\{1-\exp (-4 \tau / T)[\cos (\Delta \tau)-\Delta T / 4 \sin (\Delta \tau)]\}
$$

where $\Delta=\omega-2 \omega_{0}$ and $\omega_{0}=2 \pi \times 9 \mathrm{kHz}$, i.e., the frequency of the sound wave. This spectrum is symmetric. The observed asymmetry is attributed to the inability of the speaker to properly reproduce the phase fluctuation. 


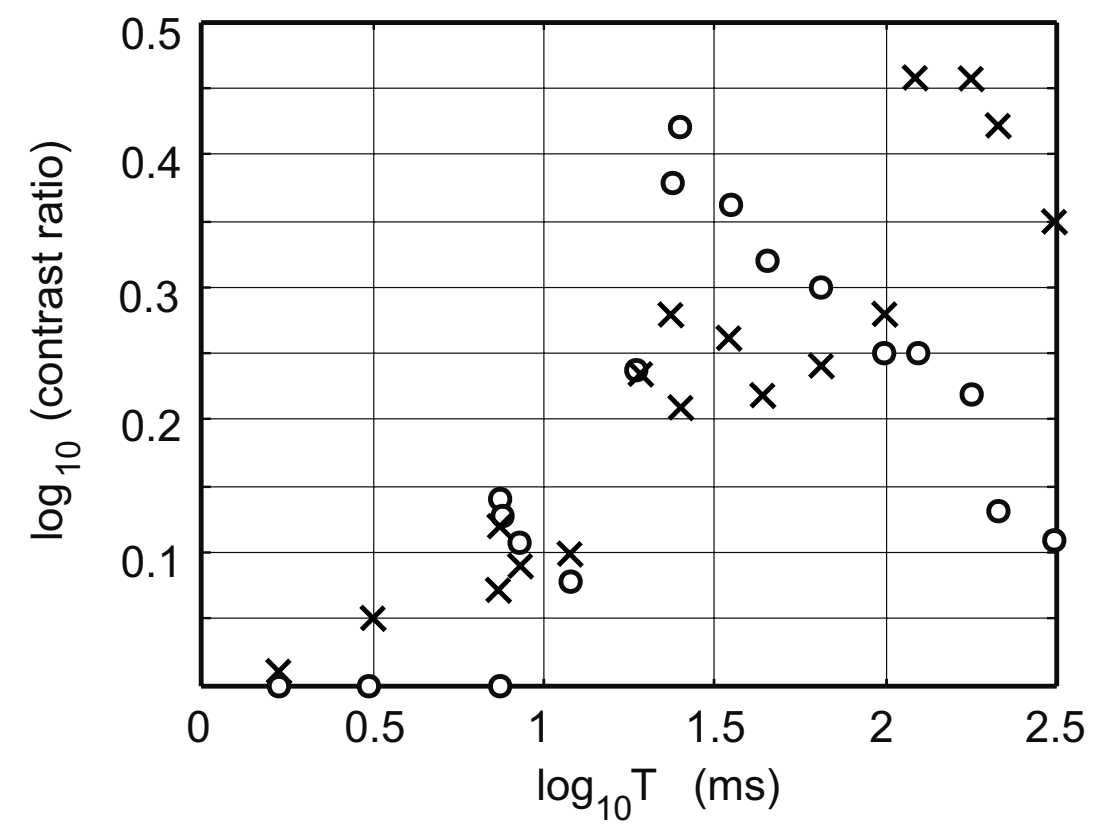

Figure 3. The ratios (expressed logarithmically) between the maxima and nearest minima for the two fringes (see Fig. 2) at $14.7 \mathrm{kHz}$ (circles) and $16.8 \mathrm{kHz}$ (crosses) as a function of the random telegraph dwell time $T$. The values of $\tau$ and $\phi$ for all data points are the same as in Fig. 2 .

The amplitude fluctuation that results is correlated with the phase jump; the correlated amplitude and phase modulations lead to an asymmetric spectrum. The theoretical relation shown is the two photon absorption spectrum, in whose derivation the atomic spectral response plays a role that is analogous to that of a window function, often used when Fourier transforming a finite series of data. As we use no window function when using the fast Fourier transform to calculate the spectra shown in Fig. 2, we must consider the two photon absorption spectrum in the limit where the width of the atomic response tends to zero in order to arrive at equation (3).

The key evidence that the fringes shown in Fig. 2 are those associated with $G^{(4)}(t, t+\tau ; \tau, 0)$ is that the fringe contrast reaches a maximum at finite $T$. Rather arbitrarily we choose two maxima (at $14.7 \mathrm{kHz}$ and $16.8 \mathrm{kHz}$ ) and the minima immediately to the high frequency side of these maxima. The ratio of maximum to minimum can be read off graphs such as Fig. 2, and the logarithm of these ratios is shown as a function of $\log _{10} T$ in Fig. 3. It is apparent that the coherence time for maximum fringe contrast depends on the distance of the fringe from the main peak at $18 \mathrm{kHz}$. The contrast maximum of the fringe at $16.8 \mathrm{kHz}$ is not seen as clearly as that for the fringe at $14.7 \mathrm{kHz}$ because in the current apparatus we have an upper limit on $T$ of about $300 \mathrm{~ms}$. The random telegraph voltage noise source incorporates a photomultiplier which detects photons from a small incandescent bulb. The detection of a photon triggers a phase jump in the voltage output which then is used as the modulation input for the HP3325. The dark current of this photomultiplier sets the upper limit on $T$. In any case, larger values of $T$ would require much more data because the time series data sets must be long enough to incorporate some phase jumps, and also there should be enough data sets to provide some averaging. We can, however, simply turn off the random telegraph noise source so that $T$ is effectively infinite. The result of this is shown in the inset of Fig. 2. The fringes vanish. In order to test that the assumption of stationarity for the stochastic process was valid, we explicitly calculated the four-time fourth-order correlation function for several consecutive subsets of many full data sets (i.e. the time series for given dwell times). The correlation functions from the subsets were essentially the same, and that the average of their Fourier transforms was indistinguishable from the function $W$ calculated in the manner described above. This is as one would expect if the correlation functions were stationary.

We have confirmed the existence of the fringes for other values of $\phi$, but we concentrate on the case of $\phi=\pi$ 
because in this case the (first order) coherence is to able to vary from complete to nonexistent. Random telegraph phase modulation results in the modulated wave having a power spectrum consisting of a delta function carrier component and a broadband Lorentzian shaped component. This is ${ }^{16}$

$$
S(\omega)=\cos ^{2}\left(\frac{\phi}{2}\right) \delta\left(\omega-\omega_{0}\right)+\sin ^{2}\left(\frac{\phi}{2}\right) \frac{4 / T}{(4 / T)^{2}-\left(\omega-\omega_{0}\right)^{2}}
$$

The width of the broadband component is inversely proportional to $T$. The relative amounts of power in these components varies with $\phi$; for $\phi=\pi$ all of the power is in the broadband component, and the coherent component is completely suppressed. ${ }^{16}$ At other values of $\phi$ a coherent component always remains, regardless of how short the dwell time $T$ might be. Curiously, it was predicted ${ }^{15}$ that for two photon absorption $\phi=\pi / 2$ would give fringes with best observability (i.e. high contrast and with good signal relative to that at line centre), but in the acoustic experiment this seems not to be the case. For a given value of $T$, the fringes are only visible over a rather restricted range in $\tau$. This however requires more thorough investigation.

\section{SECOND ORDER FRINGES FROM GPS TRANSMISSIONS}

Waves modulated with another type of "noise", pseudorandom binary noise, are readily available as transmissions from GPS satellites. The two transmission frequencies L1 $(1.57 \mathrm{GHz})$ and L2 $(1.23 \mathrm{GHz})$ are phase modulated by pseudorandom binary code sequences (prn's), which flip the phase by $\pi$ radians at zero crossings or extrema of the carrier wave. If one were to monitor the signals from a given satellite at two different locations on the earth, the difference in the path lengths between the satellite and each of the ground stations can be obtained by multiplying the respective signals (recorded simultaneously) and looking for fourth order fringes in the Fourier transform of that product. One would of course need to know the orbital parameters of the satellite, but in principle one does not need to know the prn. This scheme is complicated somewhat by the fact that a typical GPS receiver receives signals from several satellites simultaneously. The pseudorandom noise sequences are extracted by generating the prn's in the receiver and correlating them with the received signal, which is frequency down-converted, so as to identify the satellites. The beginning of each modulation sequence is synchronised to the satellite's onboard clock, so that from this correlation the signal propagation time and thus the "pseudorange" can be calculated. (The pseudorange must be corrected for various errors such as ionospheric propagation delay, clock offsets etc. to get the true range.) The originally modulated signal is not preserved in this process and would need to be reconstructed.

A proof-of-principle simulation showing that such a path difference measurement is feasible is presented in Fig. 4. The coarse acquisition (C/A) and precise (P) prn's are openly available (although the latter is rather long) and can be calculated given the prn number of the satellite. ${ }^{17}$ For Fig. 4 the $\mathrm{C} / \mathrm{A}$ code was used with the prn number 31. A reconstructed (frequency downconverted) signal with a somewhat arbitrarily chosen carrier frequency of $20.861 \mathrm{MHz}$ was produced. This signal was replicated with a small delay (500ns), and then the delayed and undelayed versions were multiplied and Fourier transformed. The modulus squared of the transform is shown in Fig. 4. This time delay corresponds to a path difference of about $150 \mathrm{~m}$, such as two GPS receivers about $260 \mathrm{~m}$ apart would see if the satellite were at an elevation of 45 degrees, and in a direction given by a line drawn through both receivers. From information such as this, and knowing the orbital parameters of the satellite, the location of one receiver with respect to the other can be determined. There is then the possibility that such measurements might supplement differential GPS techniques which use a receiver at a reference location to locate a second, possibly mobile, receiver.

There are two limits to the maximum path difference that can be detected from the second order fringes shown in Fig. 4. The first (and more severe) is the clock frequency of the pseudorandom noise generator, which gives the so-called "chipping rate". The binary digits are called chips rather than bits because they carry no information directly and the chipping rate is the rate at which chips are produced. For the C/A code this is $1.023 \mathrm{MHz}$. The binary nature of the chips and the quantisation of their duration produce dips in a spectrum such as in Fig. 4, much as would a simple square wave modulation of the phase (which would correspond to a chip sequence of $01010101 \ldots$. . These dips are spaced by $1.023 \mathrm{MHz}$ and indeed these dominate the spectrum when the delay becomes greater than 1000ns. A second limitation which is less relevant here is that the $\mathrm{C} / \mathrm{A}$-code 


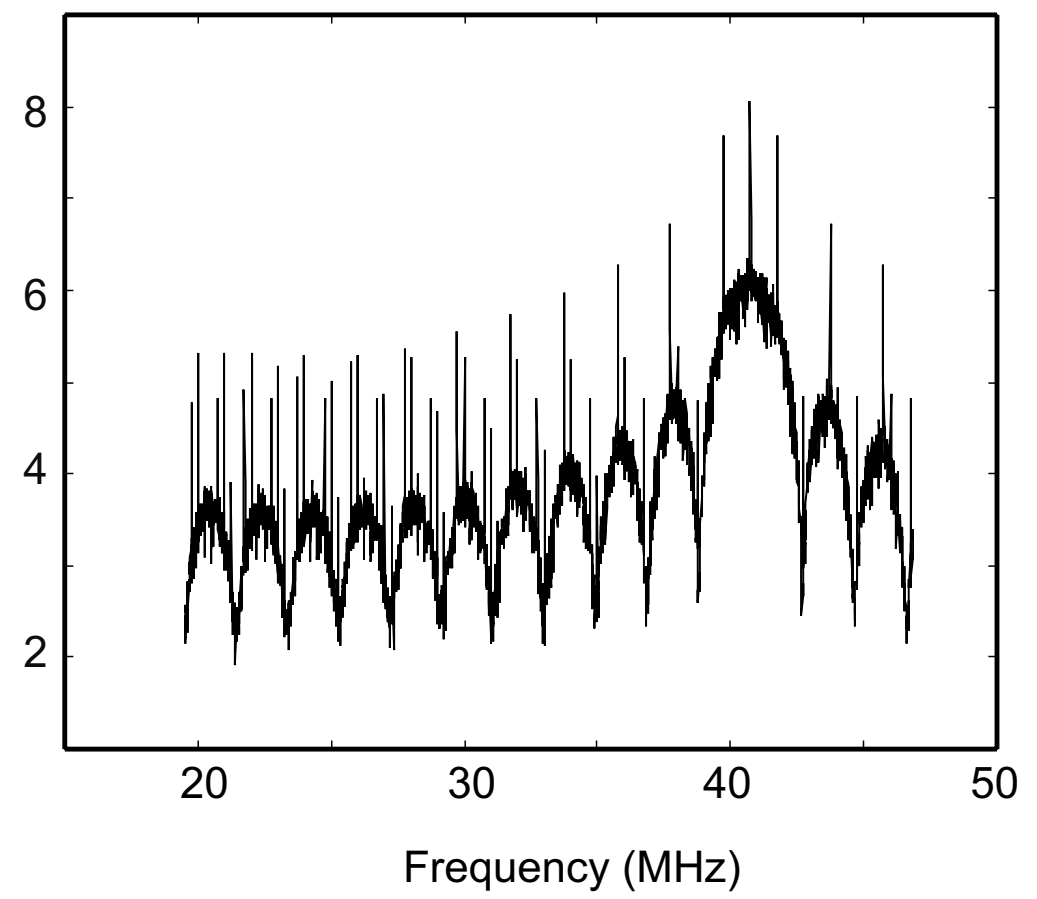

Figure 4. A simulation of second order interference fringes from the (C/A-coded) signals received by two GPS receivers with a relative time delay of $500 \mathrm{~ns}$. The highest peak is that of the $20.861 \mathrm{MHz}$ carrier frequency (multiplied by two). The fringe spacing in the wings is very close to $2 \mathrm{MHz}$, as expected from equation 3 . If the delay is halved, the fringe spacing doubles.

repeats every millisecond, so the second order fringes actually give the path difference modulo $300 \mathrm{~km}$. Both these limitations could be relaxed somewhat by use of the longer P-code with a chipping frequency of $10.23 \mathrm{MHz}$, so that the receivers could have a separation a factor of ten greater than allowed by the use of the $\mathrm{C} / \mathrm{A}$ code.

\section{DISCUSSION}

An analogy to Fraunhofer diffraction ${ }^{18}$ was developed in the context of multiphoton absorption and is of some help in interpreting our experiment. In this analogy the correlation function $G^{(4)}(t, t+\tau ; \tau, 0)$ represents the transmission function of an aperture which might be formed by a coating of varying transmission applied to a transparent substrate. The "transmission function" has sharp corners and its Fourier transform, i.e., the "diffraction pattern", will therefore show fringes. Although this analogy indicates that the fringes will exist if $G^{(4)}(t, t+\tau ; \tau, 0)$ has a sharp corner (which follows from the presence of a stochastic process) it does not, however, provide a satisfactory explanation for the maximum contrast ocurring at finite correlation time. A good heuristic explanation is lacking still.

The wave fields that we have considered so far are purely classical, but it is worthwhile to look at fields that are uniquely quantum mechanical to better put this experiment in context. It is possible to observe interference fringes in experiments with correlated photon pairs, ${ }^{8}$ where the modes into which the two photons are emitted are mixed at a beamsplitter. When the photon coincidence rate is measured between the two fields leaving the beamsplitter, interference fringes are seen as the beamsplitter is moved. This is interpreted as a first order interference of quantum probability amplitudes even though it is a second order interference measurement from an operational point of view. In contrast our experiment does not admit, in its interpretation, such an easy reduction of the order of interference, unless it is to the product of fields $E(t) E(t+\tau)$ which has no intuitively obvious significance. This is because the field fluctuations that we use here do not have a Gaussian density function. As an aside, it is well known that an ideal laser field also does not have a true Gaussian density 
function because of phase diffusion on long timescales, ${ }^{19}$ and that higher order correlations may not be reduced by the Gaussian moment theorem.

A situation that we have not considered so far is that of a field with two or more discrete frequency components. We would also expect to see, in the temporal correlation $G^{(4)}(0, t ; t, 0)$, second order fringes due to beats. Depending on the value of $t$, one detector would be sampling a node while the other might be sampling an antinode; then for a different $t$, both would be sampling nodes or antinodes simultaneously. In this case the second order fringes would not be expected to show the behaviour illustrated in Fig. 3. Rather the maximum contrast would be occur when the first order coherence is greatest, because this corresponds to the greatest visibility (or voltage standing wave ratio if it is an electromagnetic wave) of the beats. Thus that interference is fundamentally different from that which is the main subject of this paper.

\section{ACKNOWLEDGMENTS}

I would like to thank K Goldsmith and C Baker of the Defence Science and Technology Organisation for the donation of anechoic material for the acoustic experiment and for supplying the program for calculating GPS $\mathrm{C} / \mathrm{A}$ codes, and $\mathrm{K}-\mathrm{H}$ Bae for experimental assistance.

\section{REFERENCES}

1. R. Hanbury-Brown and R. Q. Twiss, "Correlation between photons in two coherent beams of light," Nature 177, p. 27, 1956.

2. M. M. Salour, "Ultra-high-resolution two-photon spectroscopy in atomic and molecular vapours," Annals of Physics 111, pp. 364-503, 1978.

3. D. S. Elliott, M. W. Hamilton, K. Arnett, and S. J. Smith, "Correlation effects of a phase diffusing laser field on two photon absorption," Phys. Rev.A 32, p. 887, 1985.

4. M. A. Dugan, J. S. Melinger, and A. C. Albrecht, "Terahertz oscillations from molecular liquids in csrs/cars spectroscopy with incoherent light," Chem. Phys. Letts, 147, pp. 411-419, 1988.

5. M. A. Dugan and A. C. Albrecht, "Radiation-matter oscillations and spectral line narrowing in fieldcorrelated four-wave mixing," Physical Review A 43, p. 3922, 1991.

6. V. Kozich, L. se S Menezes, and C. B. de Araujo, "Interference effects in time-delayed degenerate four wave mixing with broadband noisy light," J. Opt. Soc. Am. B 17, pp. 973-976, 2000.

7. M. Hamilton, "Interference fringes with maximal contrast at finite coherence time," Phys. Rev. Letts. 89, 2002.

8. L. Mandel, "Quantum effects in one-photon and two-photon interference," Reviews of Modern Physics $\mathbf{7 1}$, p. S274, 1999.

9. G. S. Agarwal, J. von Zanthier, C. Skornia, and H. Walther, "Intensity-intensity correlations as a probe of interferences under conditions of noninterference in the intensity," Physical Review A 65, p. 053826, 2002.

10. A. B. Haner and N. R. Isenor, "Intensity correlation from pseudothermal light sources," American Journal of Physics 38, pp. 748-750, 1970.

11. R. Hanbury-Brown, The Stellar Interferometer, Taylor and Francis, 1974.

12. L. Mandel and E. Wolf, Optical Coherence and Quantum Optics, Cambridge University Press, New York, 1995.

13. A. Papoulis, Probability, Random Variables and Stochastic Processes, McGraw-Hill, 1985.

14. M. W. Hamilton and G. N. Sinclair, "Some techniques for artificial spectral broadening of lasers," Rev. Sci. Instrum. 65, p. 2180, 1994.

15. M. W. Hamilton and D. S. Elliott, "Second order interference fringes in two photon absorption," Journal of Modern Optics 43, pp. $1765-1771,1996$.

16. J. H. Eberly, K. Wodkiewicz, and B. W. Shore, "Noise in strong laser-atom interactions: Phase telegraph noise," Phys. Rev. A 30, pp. 2381-2389, 1984.

17. P. Ward, Understanding GPS: Principles and Applications (Ed. E.D. Kaplan), ch. 4, pp. 83-117. Artech, Boston, 1996. 
18. B. Broers, L. D. Noordam, and H. B. van Linden van den Heuvell, "Diffraction and focussing of spectral energy in multiphoton processes," Phys. Rev. A 46, pp. 2749-2756, 1992.

19. R. Loudon, The Quantum Theory of Light, Oxford, 1973. 